\title{
Optimization of Handover Mechanism in 802.16e using Fuzzy Logic
}

\author{
Ewa Kozlowska \\ Czech Technical University of Prague, Department of Telecommunication Engineering, \\ Technicka 2, 16627 Prague 6, Czech Republic
}

kozloe1@fel.cvut.cz

\begin{abstract}
This paper describes the handover mechanism in the standard 802.16e, which has implemented three handover methods - Hard Handover (HHO), Fast Base Station Switching (FBSS) and Macro Diversity Handover (MDHO) at this moment. Moreover, the handoff scheme is presented based on fuzzy logic. The mechanism takes under consideration three criteria: geographical data, received power level and used bandwidth of each base station. Fuzzy logic has been used to come with the decision about the target BS to be chosen such that the switching between BSs is more efficient.
\end{abstract}

Keywords: handoff, fuzzy logic

\section{Introduction}

Mobility is the most important feature of a wireless cellular communication system. Undoubtedly the future perspective of networking will demand to support mobile users traveling all over the world. In addition to that, the number of portable devices that need access to the Internet is exponentially increasing. Users on the other hand are no longer required to work in their company's home network while they may be moving from place to place. Usually, continuous service is achieved by supporting handover from one cell to another. Handover is the process of changing the channel (frequency, time slot, spreading code, or combination of them) associated with the current connection while a call is in progress. It is often initiated either by crossing a cell boundary or by deterioration in quality of the signal in the current channel. Handover is divided into two broad categories - hard and soft. They are also characterized by „break before make" and "make before break." In hard handover, current resources are released before new resources are used; in soft handover, both exist and new resources are used during the handover process. Poorly designed handover schemes tend to generate very heavy signaling traffic and, thereby, a dramatic decrease in quality of service (QoS). The reason why handovers are critical in cellular communication systems is that neighboring cells are always using a disjoint subset of frequency bands, so negotiations must take place between the mobile station (MS), the current serving base station (BS), and the next potential BS. Other related issues, such as decision making and priority strategies during overloading, might influence the overall performance [1].

Please use the following format when citing this chapter:

Kozlowska, E., 2007, in IFIP International Federation for Information Processing, Volume 245, Personal Wireless Communications, eds. Simak, B., Bestak, R., Kozowska, E., (Boston: Springer), pp. 115-122. 


\section{Overview of Handover Mechanism in 802.16e}

Two crucial issues for mobile applications are battery life and handover. To enable power-efficient MS function Mobile WiMAX supports Sleep Mode and Idle Mode. 802.16e also supports seamless handover to allow the MS to switch from one base station to another at vehicular speeds without interrupting the connection. Handover schemes should be with latencies less than 50 milliseconds to ensure real-time applications such as VoIP perform without service degradation. Flexible key management schemes assure that security is maintained during handover [2].

There are three handover methods supported within the 802.16e standard: Hard Handover (HHO), Fast Base Station Switching (FBSS) and Macro Diversity Handover (MDHO). While the HHO is mandatory, FBSS and MDHO are two optional modes.

\subsection{Hard Handover (HHO)}

It begins with cell reselection proceeded by the MS. Information about adjoining BSs may be obtain from a decoded MOB_NBR-ADV message or a request to schedule scanning intervals or sleep-intervals can be sent to the serving BS. MOB_NBR-ADV message is broadcasted by the BS to identify the network and define the characteristics of the neighbour BS to the potential MS searching initial network entry or the handover. Intention of this stage is to check HHO possibilities. Procedure of searching available the neighbour BS does not involve termination of existing connection to a serving BS. Next phase is HO Decision and Initiation, which can be start either by the MS, by sending an MOB MSHO-REQ message or the serving BS, by sending an MOB_BSHO-REQ message. In case of MS initiating handover with the BS, the serving BS replies with MOB_BSHO-RSP message containing instruction to the $\mathrm{HHO}$ with neighbour BSs list. After the handover preparation ends, execution follows. The target BS is selected by the MS and MOB_HO-IND message is sent; by sending this message the MS terminates service with the serving BS. As soon as the MS synchronize with the target BS, ranging process begins. It mainly aims to time synchronization and power adjustment. Then the MS negotiates basic capabilities, performs authentication and finally registers with the target BS. Whole this process can be shortened, if the target BS learns MS information from serving BS thru backbone network. Cancellation of the HHO is possible at any time till the expiration of Resource_Retain_Time interval after transmission of MOB_HO-IND message [3].

\subsection{Macro Diversity Handover (MDHO)}

Support of MDHO is optional for both the MS and the BS. The MS and the BS should hold a list of BSs involved in MDHO with the MS. The list name is Diversity Set. One of BSs in the Diversity Set is defined as an Anchor BS. Decision about performing handover in MDHO begins with an MS transmitting/receiving unicast message and traffic from multiple BSs at the same time interval. For downlink $\mathrm{MDHO}$, two or more BSs provide synchronized transmission of MS downlink data 
such that diversity combining is performed at the MS. For uplink MDHO, the transmission from a MS is received by multiple BSs where selection diversity of the information received is performed. [3].

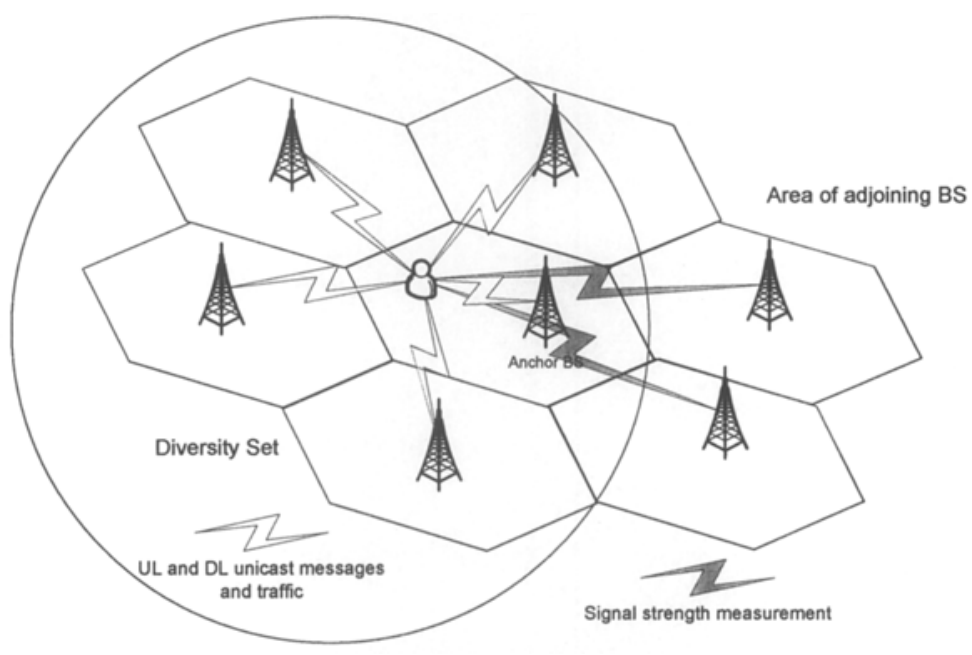

Fig. 1. Macro Diversity Handover.

\subsection{Fast Base Station Switching (FBSS)}

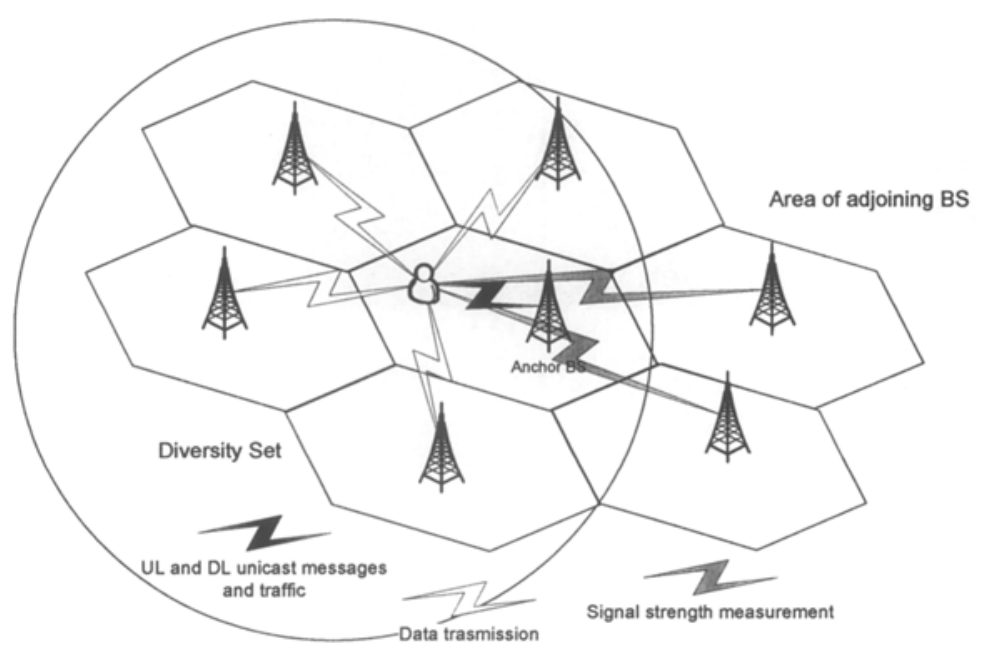

Fig. 2. Fast Base Station Switching.

Support of FBSS is optional for both the MS and the BS. Also in this mechanism the MS and the BS possess a list of BSs that are taking part in FBSS with the MS. The name of the list is exactly the same like in case of MDHO, Diversity Set and also the 
Anchor BS is defined. In FBSS, the MS continuously monitors the base stations in the Diversity Set. FBSS begins with decision for an MS to receive/transmit data from/to the Anchor BS that may change within the Diversity Set. Switch from one Anchor BS to another is performed without proceeding $\mathrm{HO}$ signaling message. Scanning to search for suitable adjoining BS to update the set is made by the MS. By endless monitoring of the Diversity Set BSs signal strength, the MS is capable of choosing one as the Anchor BS. This selection should be reported by the MS to the network. An important requirement of FBSS is that the data is simultaneously transmitted to all members of an active set of BSs that are able to serve the MS [3].

\section{$2.4 \quad$ Updating}

Update of diversity se depends on the threshold contained in Downlink Channel Descriptor (DCD). Two thresholds are defined: $\mathrm{H}_{-}$Delete threshold and $\mathrm{H}_{-}$Add threshold. In case of dropping the serving BS from Diversity Set, the long-term CINR of serving BS has to be smaller than $\mathrm{H}_{-}$Delete Threshold. In case of adding a new BS to Diversity Set, the logn-term CINR of neighbour BS is bigger than H_Add Threshold. For both MDHO and FBSS the Diversity Set update is performed exactly the same.

Updating of Anchor BS is solved by two mechanisms. The first, called „Handover MAC Management Method", is based on exchanging five types of MAC management messages. The second method, noted as „Fast Anchor BS Selection Mechanism”, uses Fast Feedback channel for exchanging anchor Bs selection information. A new anchor BS choice is made according to signal strength measurement reported by the MS. A new anchor BS should be included in the present Diversity Set [4].

\section{Overview of Fuzzy Logic}

Fuzzy Logic is a problem-solving control system methodology that lends itself to implementation in systems ranging from simple, small, embedded micro-controllers to large, networked, multi-channel PC or workstation-based data acquisition and control systems. It can be implemented in hardware, software, or a combination of both. FL provides a simple way to arrive at a definite conclusion based upon vague, ambiguous, imprecise, noisy, or missing input information. FL's approach to control problems mimics how a person would make decisions, only much faster.

FL requires some numerical parameters in order to operate such as what is considered significant error and significant rate-of-change-of-error, but exact values of these numbers are usually not critical unless very responsive performance is required in which case empirical tuning would determine them.

The fuzzy logic analysis and control method is, therefore:

1. Receiving of one, or a large number, of measurement or other assessment of conditions existing in some system we wish to analyze or control.

2. Processing all these inputs according to human based, fuzzy "If-Then" rules, which can be expressed in plain language words, in combination with traditional non-fuzzy processing. 
3. Averaging and weighting the resulting outputs from all the individual rules into one single output decision or signal which decides what to do or tells a controlled system what to do. The output signal eventually arrived at is a precise appearing, defuzzified, "crisp" value.

The following is Fuzzy Logic Control/Analysis Method diagram:

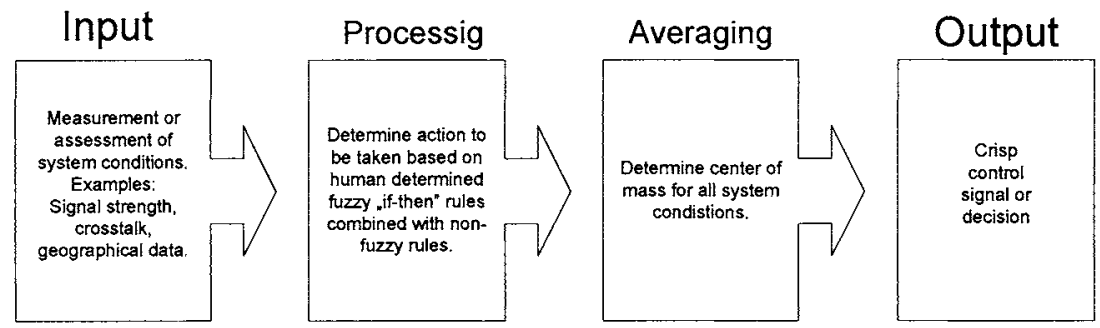

Fig. 3. The fuzzy logic Control-Analysis method.

Fuzzy Logic provides a completely different, unorthodox way to approach a control problem. This method focuses on what the system should do rather than trying to understand how it works. One can concentrate on solving the problem rather than trying to model the system mathematically, if that is even possible. This almost invariably leads to quicker, cheaper solutions. Once understood, this technology is not difficult to apply and the results are usually quite surprising and pleasing [5].

\section{Proposed Optimization of Handover in 802.16e}

In the fully mobile scenario, user expectations for connectivity are comparable to those experienced in $3 \mathrm{G}$ voice/data systems. Users may be moving while simultaneously engaging in a broadband data access or multimedia streaming session. The need to support low-latency and lowpacket-loss handovers of data streams as users transition from one BS to another is clearly a challenging task. For mobile data services, users will not easily adapt their service expectations because of environmental limitations that are technically challenging but not directly relevant to the user (such as being stationary or moving). For these reasons, the network and air interface must be designed upfront to anticipate these user expectations and deliver accordingly [6].

The one of idea in proposed mechanism is to predict movement of mobile user, by constructing an intelligent map of terrain with use of geographical data gathered from mobile users and base stations. Base stations position is fixed as there is no movement. An idea of using GPS receiver to provide the most accurate and robust source of time and frequency synchronization for WiMAX networks has been already given. Those receivers can be also use to determine geographical position of base station. As far as it goes for the mobile user, it is required to have implemented GPS receiver, which features we can use to create an intelligent map of terrain. Only three ways of movement we are interested in: 
- by train, where destination point is known and the route is fixed,

- by car, where destination point is known and the route is predictable,

- on foot, where a velocity of mobile user is small, and by this predictable.

The main idea of presented handoff scheme is based on the fuzzy logic. The schemes consider not only received power level criterion, but also the bandwidth (data transfer rate in Kbps) and distance from the base station. Fuzzy logic here is used to reach a decision about the target BS to be chosen such that the handoff is performed as fast as possible with reasonable QoS levels.

I phase. First phase of fuzzy logic proceeding is to deliver input parameters of signal strength, distance from the base station and bandwidth use in the cell. Following parameters are fuzzified with use of pre-defined input membership functions, for example, membership function for distance shown on the figure Fig. 4:

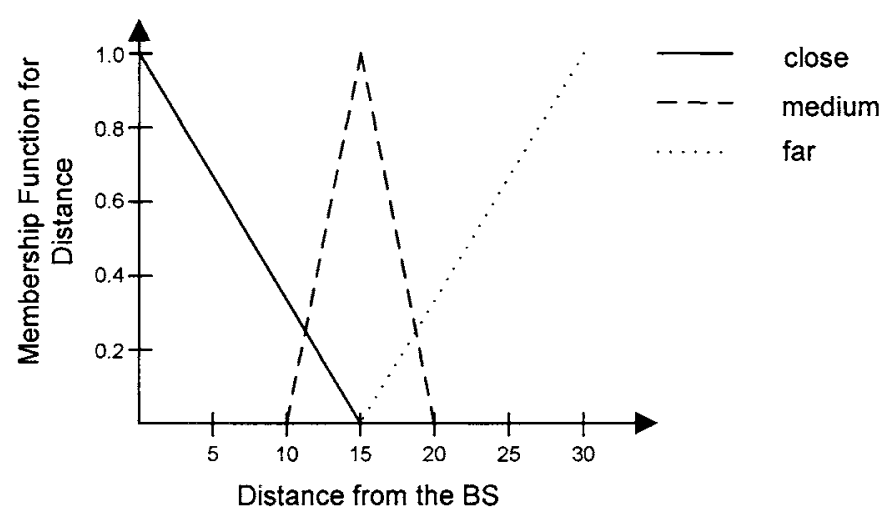

Fig. 4. Input membership function of distance from a BS.

As example triangular member function has been shown, but other shapes can also be chosen if their representation of real scenario in the 802.16e network is more suitable. The first step of the fuzzy logic Control-Analysis method is to transform the input value to names of member function and degrees of memberships in the function. For instance, given distance from $\mathrm{BS}=14$, the following value can be read off the graph from Fig. 4.

Input

Distance From BS (=14)

(Membership-Fn, Membership-Degree)

$(\mathrm{C}, 0.1) \quad(\mathrm{M}, 0.75)$

where, $\mathrm{C}$ - close, $\mathrm{M}$ - medium, $\mathrm{F}$ - far distance.

Second phase. Here the rule matrix is used, which has two input conditions, "error" and "error-dot" (rate-of-change-of-error), and one output response conclusion (at the intersection of each row and column). The rule matrix is a simple graphical tool for mapping the FL control system rules. It accommodates two input variables and expresses their logical product (AND) as one output response variable. The degree of membership for rule matrix output can take value of maximum, minimum or the average of the degree of previous of the rule [7]. It is often probable, that after 
evaluation of all the rules applicable to the input, we get more than one value for the degree of membership. In this case, the simulation has to take under consideration, all three possibilities, the minimum, maximum or average of the membership-degrees.

Third phase. It can be called defuzzification. The defuzzification of the data into a crisp output is accomplished by combining the results of the inference process and then computing the "fuzzy centroid" of the area. The weighted strengths of each output member function are multiplied by their respective output membership function center points and summed. Finally, this area is divided by the sum of the weighted member function strengths and the result is taken as the crisp output. One feature to note is that since the zero center is at zero, any zero strength will automatically compute to zero [7].

In fuzzy handoff decision mechanism, to the truth values have to have assigned a set of weights, represented by table of weight. According to these fuzzy handoff decision weight values and the degree of membership for fuzzy handoff decision output, the crisp value of output is determined by following formula:

$$
\text { fuzzy_handoff_decisin }=\frac{\sum \mathrm{M}_{\mathrm{i}} \times \mathrm{W}_{\mathrm{i}}}{\sum \mathrm{M}_{\mathrm{i}}}
$$

where, $M_{i}$ is the degree of membership in output singleton $i$, and $W_{i}$ is the fuzzy handoff decision weight value for the output singleton $i$.

The crisp value of fuzzy logic decision is computed for each of the adjacent base stations. In result, the BS with highest value of decision is chosen as a target BS.

\section{Conclusions}

The theme presented in this paper is a part of the research work being done as the first phase of a study of handoff techniques optimization in standard 802.16e. We are currently working on the simulation model, to carry out simulation for all procedures, min-min, min-max, min-avg, max-min, max-max, max-avg, avg-min, avg-max, and avg-avg, in order to investigate the effect of the function choice on the result of the fuzzy logic process.

The handoff mechanism based on fuzzy logic presented in this paper hopefully will result in more efficient switching between BSs. Therefore, it can be used to provide sufficient QoS and better response time to handover request. Fuzzy logic handover scheme, by use of various input sets, is flexible and probably will give good results, even with wide set of input conditions. Proceeding based on fuzzy logic can be easily implemented through uncomplicated software procedures or dedicated fuzzy logic processing modules.

Acknowledgement. The article was supported by Czech Technical University's grant No. CTU0715013. 


\section{References}

1. http://en.wikipedia.org/wiki/Handover [29.03.2007]

2. WiMAX Forum, Mobile WiMAX - Part I: A Technical Overview and Performance Evaluation, June, 2006

3. IEEE 802.16e-2005: Air Interface for Fixed and Mobile Broadband Wireless Access Systems, p. 244-257, February 2006

4. Bečvár, Z. and Zelenka, J.: Handovers in the Mobile WiMAX, In Research in Telecommunication Technology 2006 - Proceedings [CD-ROM]. Brno: Vysoké učení technické v Brně, 2006, vol. I, s. 147-150. ISBN 80-214-3243-8.

5. http://www.fuzzy-logic.com/Ch1.htm [04.03.2007]

6. Deepak Pareek: The business of WiMAX, John Wiley \& Sons Ltd, 2006

7. http://www.seattlerobotics.org/encoder/mar98/fuz/flindex.html [04.03.2007] 